\section{Crystal structure of cis-tetraaqua- dichloridocobalt(II) sulfolane disolvate}

\author{
Mhamed Boudraa, ${ }^{\mathrm{a}}$ Sofiane Bouacida, ${ }^{\mathrm{a}, \mathrm{b}}{ }$ Hasna \\ Bouchareb, ${ }^{a}$ Hocine Merazig ${ }^{\mathrm{a}}$ and El Hossain Chtoun ${ }^{\mathrm{c}}$ \\ aUnité de Recherche de Chimie de l'Environnement et Moléculaire Structurale, \\ CHEMS, Université Constantine 1, 25000, Algeria, 'bépartement Sciences de la \\ Matière, Faculté des Sciences Exactes et Sciences de la Nature et de la Vie, Université \\ Oum El Bouaghi, Algeria, and ' ${ }^{\mathrm{C}}$ niversité Abdelmalek Essaadi, Faculté des Sciences, \\ BP 2121 M'Hannech II, 93002 Tétouan, Morocco. *Correspondence e-mail: \\ bouacida_sofiane@yahoo.fr
}

Received 7 December 2014; accepted 19 December 2014

Edited by W. Imhof, University Koblenz-Landau, Germany

In the title compound, $\left[\mathrm{CoCl}_{2}\left(\mathrm{H}_{2} \mathrm{O}\right)_{4}\right] \cdot 2 \mathrm{C}_{4} \mathrm{H}_{8} \mathrm{SO}_{2}$, the $\mathrm{Co}^{\mathrm{II}}$ cation is located on the twofold rotation axis and is coordinated by four water molecules and two adjacent chloride ligands in a slightly distorted octahedral coordination environment. The cisoid angles are in the range 83.27(5)$99.66(2)^{\circ}$. The three transoid angles deviate significantly from the ideal linear angle. The crystal packing can be described as a linear arrangement of complex units along $c$ formed by bifurcated $\mathrm{O}-\mathrm{H} \cdots \mathrm{Cl}$ hydrogen bonds between two water molecules from one complex unit towards one chloride ligand of the neighbouring complex. Two solvent molecules per complex are attached to this infinite chain via $\mathrm{O}-\mathrm{H} \cdots \mathrm{O}$ hydrogen bonds in which water molecules act as the hydrogenbond donor and sulfolane $\mathrm{O}$ atoms as the hydrogen-bond acceptor sites.

Keywords: crystal structure; cobalt(II) complex; sulfolane solvate.

CCDC reference: 1040554

\section{Related literature}

For structures where the $\mathrm{Co}^{\mathrm{II}}$ atom exhibits an octahedral geometry and is coordinated by water molecules, see: Waizumi et al. (1990); Sarangarajan et al. (2008). For potential applications of organic-inorganic hybrid compounds, see: AlKtaifani \& Rukiah (2011). For related structures, see: Bouacida et al. (2005, 2013); Sahbani et al. (2014).
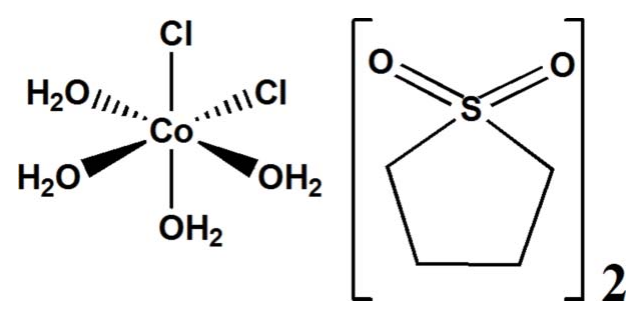

\title{
2. Experimental
}

\subsection{Crystal data \\ $\left[\mathrm{CoCl}_{2}\left(\mathrm{H}_{2} \mathrm{O}\right)_{4}\right] \cdot 2 \mathrm{C}_{4} \mathrm{H}_{8} \mathrm{O}_{2} \mathrm{~S}$ \\ $M_{r}=442.22$ \\ Monoclinic, $C 2 / c$ \\ $a=20.062(2) \AA$ \\ $b=9.4284(10) \AA$ \\ $c=10.5882(13) \AA$ \\ $\beta=118.734(5)^{\circ}$}

\subsection{Data collection \\ Bruker APEXII diffractometer \\ Absorption correction: multi-scan (SADABS; Sheldrick, 2002) \\ $T_{\min }=0.649, T_{\max }=0.748$}

\subsection{Refinement}

$R\left[F^{2}>2 \sigma\left(F^{2}\right)\right]=0.043$

$w R\left(F^{2}\right)=0.129$

$S=1.01$

5090 reflections

108 parameters

4 restraints

Table 1

Hydrogen-bond geometry $\left(\AA,^{\circ}\right)$.

\begin{tabular}{lllll}
\hline$D-\mathrm{H} \cdots A$ & $D-\mathrm{H}$ & $\mathrm{H} \cdots A$ & $D \cdots A$ & $D-\mathrm{H} \cdots A$ \\
\hline $\mathrm{O} 1 W-\mathrm{H} 1 W \cdots \mathrm{Cl} 1^{\mathrm{i}}$ & $0.77(3)$ & $2.44(3)$ & $3.1885(15)$ & $165(3)$ \\
$\mathrm{O} 1 W-\mathrm{H} 2 W \cdots \mathrm{O} 11$ & $0.81(2)$ & $1.99(2)$ & $2.782(2)$ & $165(2)$ \\
$\mathrm{O} 2 W-\mathrm{H} 3 W \cdots \mathrm{C} 1^{\mathrm{ii}}$ & $0.83(2)$ & $2.41(2)$ & $3.2289(16)$ & $171(2)$ \\
$\mathrm{O} 2 W-\mathrm{H} 4 W \cdots \mathrm{O} 12$ & $0.79(3)$ & $2.05(3)$ & $2.835(2)$ & $174(3)$ \\
\hline
\end{tabular}

Symmetry codes: (i) $-x+1,-y+1,-z$; (ii) $x,-y+1, z+\frac{1}{2}$.

Data collection: APEX2 (Bruker, 2011); cell refinement: SAINT (Bruker, 2011); data reduction: $S A I N T$; $\operatorname{program}(\mathrm{s})$ used to solve structure: SIR2002 (Burla et al., 2005); program(s) used to refine structure: SHELXL97 (Sheldrick, 2008); molecular graphics: ORTEP-3 for Windows (Farrugia, 2012) and DIAMOND (Brandenburg \& Berndt, 2001); software used to prepare material for publication: Win $G X$ (Farrugia, 2012).

\section{Acknowledgements}

Thanks are due to MESRS and ATRST (Ministére de l'Enseignement Supérieur et de la Recherche Scientifique et l'Agence Thématique de Recherche en Sciences et Technologie - Algérie) for financial support via the PNR programme. 
Supporting information for this paper is available from the IUCr electronic archives (Reference: IM2458).

\section{References}

Al-Ktaifani, M. M. \& Rukiah, M. K. (2011). Chem. Pap. 65, 469-476.

Bouacida, S., Bouchene, R., Khadri, A., Belhouas, R. \& Merazig, H. (2013). Acta Cryst. E69, m610-m611.

Bouacida, S., Merazig, H., Beghidja, A. \& Beghidja, C. (2005). Acta Cryst. E61, m577-m579.

Brandenburg, K. \& Berndt, M. (2001). DIAMOND. Crystal Impact, Bonn, Germany.
Bruker (2011). APEX2 and SAINT. Bruker AXS Inc., Madison, Wisconsin, USA.

Burla, M. C., Caliandro, R., Camalli, M., Carrozzini, B., Cascarano, G. L., De Caro, L., Giacovazzo, C., Polidori, G. \& Spagna, R. (2005). J. Appl. Cryst. 38, 381-388.

Farrugia, L. J. (2012). J. Appl. Cryst. 45, 849-854.

Sahbani, T., Smirani Sta, W. \& Rzaigui, M. (2014). Acta Cryst. E70, m6.

Sarangarajan, T. R., Krishnamoorthy, B. S., Panchanatheswaran, K., Low, J. N. \& Glidewell, C. (2008). Acta Cryst. C64, m286-m291.

Sheldrick, G. M. (2002). SADABS. University of Göttingen, Germany.

Sheldrick, G. M. (2008). Acta Cryst. A64, 112-122.

Waizumi, K., Masuda, H., Ohtaki, H., Tsukamoto, K. \& Sunagawa, I. (1990). Bull. Chem. Soc. Jpn, 63, 3426-3433. 


\section{supporting information}

Acta Cryst. (2015). E71, m16-m17 [doi:10.1107/S2056989014027753]

\section{Crystal structure of cis-tetraaquadichloridocobalt(II) sulfolane disolvate}

\section{Mhamed Boudraa, Sofiane Bouacida, Hasna Bouchareb, Hocine Merazig and El Hossain Chtoun}

\section{S1. Comment}

Organic-inorganic hybrid salts have received considerable attention because of their potential applications in analytical, material and supramolecular chemistry (Al-Ktaifani et al., 2011; Bouacida et al. 2005, 2013; Sahbani et al., 2014). In this work, we report the preparation and the structural investigation of $\left[\mathrm{Co}\left(\mathrm{H}_{2} \mathrm{O}\right)_{4} \mathrm{Cl}_{2}\right] \times 2 \mathrm{C}_{4} \mathrm{H}_{8} \mathrm{SO}_{2}$. Since the central cobalt the $\mathrm{Co}^{\mathrm{II}}$ cation is located on the twofold rotation axis the asymmetric unit of (I) consists of one one half of the complex unit and one molecule of sulfolane (Figure 1).

The structure of the compound consists of discrete tetraaquadichlorocobalt(II) complexes stacked in chains parallel to the $c$ axis. The $\mathrm{Co}^{\mathrm{II}}$ cation is coordinated by four water molecules and two adjacent chloride ligands in a slightly distorted octahedral geometry. The two $\mathrm{Co}-\mathrm{Cl}$ distances are 2.510 (6) $\AA$ and the $\mathrm{Co}-\mathrm{O}$ distances are between 2.165 (3) and 2.243 (3) $\AA$ in good agreement with that found in mineral compound $\mathrm{CoCl}_{2} \mathrm{O}_{4} \mathrm{H}_{8}$ (Waizumi et al., 1990) and in the coordination compound $\left[\mathrm{CoCl}_{2}\left(\mathrm{H}_{2} \mathrm{O}\right)_{4}\right] \mathrm{C}_{4} \mathrm{H}_{6} \mathrm{~N}_{2} \mathrm{O}_{2}$ (Sarangarajan et al., 2008). Cisoid angles around Co atom are in the range of $83.27(5)^{\circ}$ to $99.66(2)^{\circ}$. In the organic molecule, the $\mathrm{S}$ atom is tetrahedrally coordinated by two $\mathrm{O}$ and two $\mathrm{C}$ atoms. The three bonds $\mathrm{C}-\mathrm{C}$ are in the range 1.516 (2) $-1,531$ (3) $\AA$. In the crystal, molecules are linked by $\mathrm{O}-\mathrm{H} \cdots \mathrm{O}$ and $\mathrm{O}-\mathrm{H} \cdots \mathrm{Cl}$ hydrogen bonds forming chains along [001] (Figure 2). The crystal packing can be described as a linear arrangement of complex units along $c$ formed by bifurcated $\mathrm{O}-\mathrm{H} \cdots \mathrm{Cl}$ hydrogen bonds between two water molecules from one complex unit towards one chloride ligand of the neighboring complex. Two solvent molecules per complex are attached to this infinite chain via $\mathrm{O}-\mathrm{H} \cdots \mathrm{O}$ hydrogen bonds in which water molecules act as the hydrogen bond donor and sulfolane oxygen atoms as the hydrogen bond acceptor sites.

\section{S2. Experimental}

A solution of $\mathrm{CoCl}_{2} \times 2 \mathrm{H}_{2} \mathrm{O}(34 \mathrm{mg}, 0.2 \mathrm{mmol})$ in water $(10 \mathrm{ml})$ was added dropwise to a solution of sulfolane $(24 \mathrm{mg}$, $0.2 \mathrm{mmol}$ ) in water $(10 \mathrm{ml})$. The mixture was then refluxed with stirring for $3 \mathrm{~h}$ and the resulting solution was left to stand at room temperature. After several days, blue crystals were obtained and dried under vacuum (yield: 55\%).

\section{S3. Refinement}

All non-H atoms were refined with anisotropic displacement parameters. Approximate positions for all $\mathrm{H}$ atoms were first obtained from the difference electron density map. However, the $\mathrm{H}$ atoms were situated into idealized positions and the $\mathrm{H}$-atoms have been refined within the riding atom approximation with $\mathrm{C}-\mathrm{H}=0.93 \AA$ and $U_{\text {iso }}=1.2 U_{\text {eq }}(\mathrm{C})$ except for $\mathrm{H}$ atoms of water molecules, which were refined isotropically using the following restraints: $\mathrm{O}-\mathrm{H}=0.84$ (2) $\AA, \mathrm{H} \cdots \mathrm{H}=$ $1.45(2) \AA ̊$ and $U_{\text {iso }}=1.5 U_{\text {eq }}(\mathrm{O})$. 


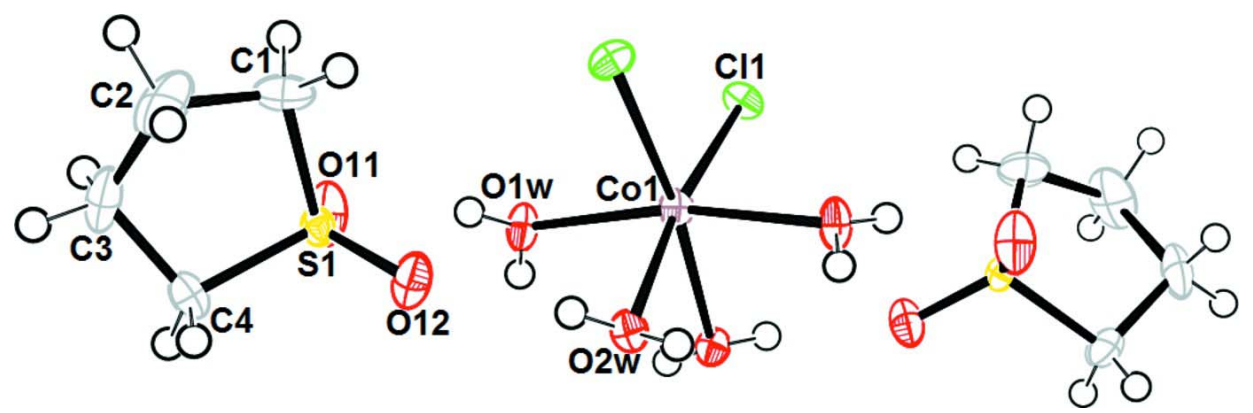

Figure 1

Molecular structure of (I) with displacement ellipsoids drawn at the $50 \%$ probability level. Only the asymmetric unit is labelled. $\mathrm{H}$ atoms are represented as small spheres of arbitrary radii.

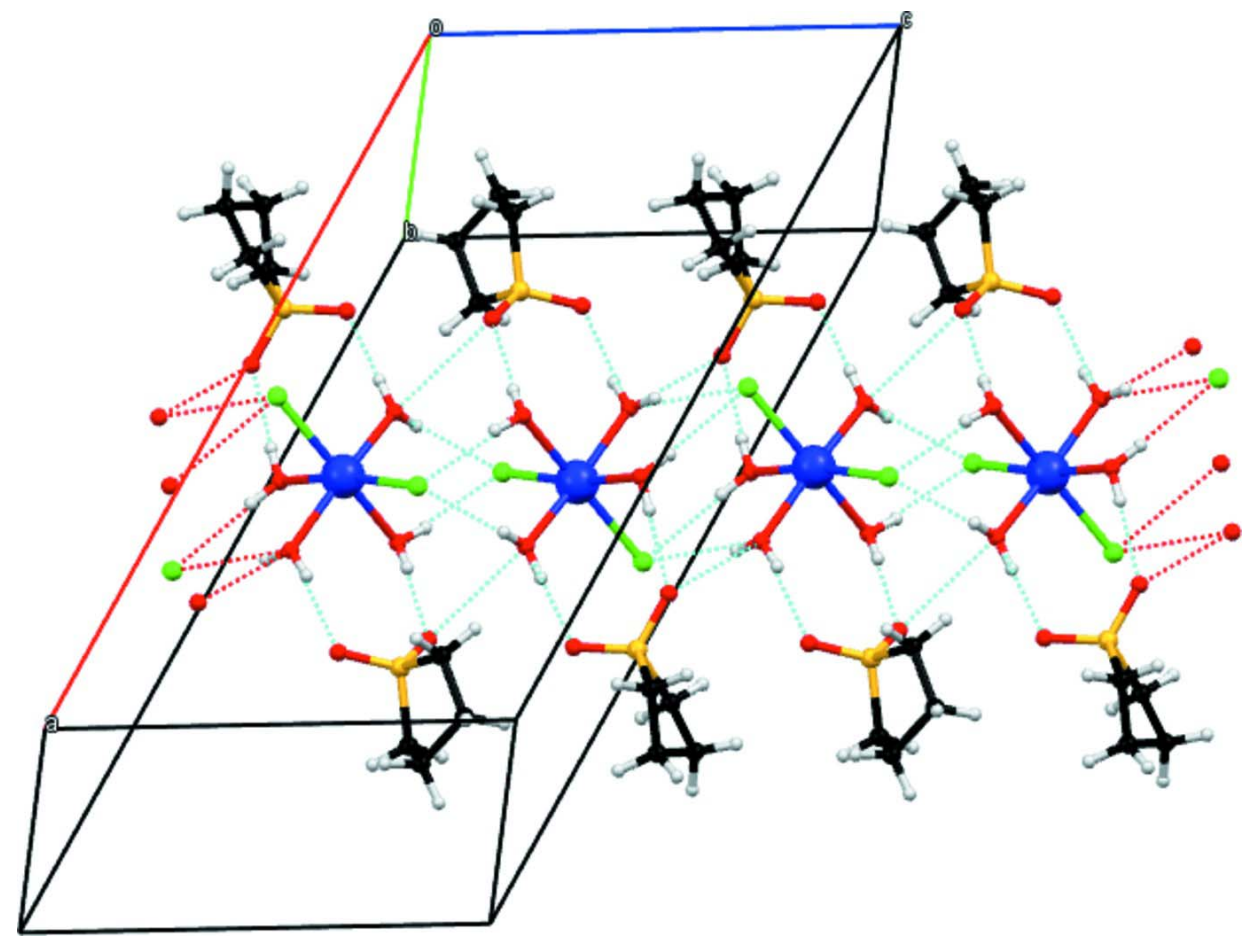

Figure 2

Packing diagram of (I) showing the infinite chains of complex units and solvent molecule along the $c$ axis.

cis-Tetraaquadichloridocobalt(II) sulfolane disolvate

Crystal data

$\left[\mathrm{CoCl}_{2}\left(\mathrm{H}_{2} \mathrm{O}\right)_{4}\right] \cdot 2 \mathrm{C}_{4} \mathrm{H}_{8} \mathrm{O}_{2} \mathrm{~S}$

$M_{r}=442.22$

Monoclinic, $C 2 / c$

Hall symbol: $-\mathrm{C} 2 \mathrm{yc}$

$a=20.062(2) \AA$

$b=9.4284(10) \AA$

$c=10.5882(13) \AA$

$\beta=118.734(5)^{\circ}$

$V=1756.2(3) \AA^{3}$

$Z=4$
$F(000)=916$

$D_{\mathrm{x}}=1.673 \mathrm{Mg} \mathrm{m}^{-3}$

Mo $K \alpha$ radiation, $\lambda=0.71073 \AA$

Cell parameters from 5148 reflections

$\theta=2.5-35.1^{\circ}$

$\mu=1.55 \mathrm{~mm}^{-1}$

$T=295 \mathrm{~K}$

Prism, blue

$0.21 \times 0.15 \times 0.09 \mathrm{~mm}$ 


\section{Data collection \\ Bruker APEXII \\ diffractometer \\ Radiation source: sealed tube \\ Graphite monochromator \\ $\varphi$ and $\omega$ scans \\ Absorption correction: multi-scan \\ (SADABS; Sheldrick, 2002) \\ $T_{\min }=0.649, T_{\max }=0.748$}

\section{Refinement}

Refinement on $F^{2}$

Least-squares matrix: full

$R\left[F^{2}>2 \sigma\left(F^{2}\right)\right]=0.043$

$w R\left(F^{2}\right)=0.129$

$S=1.01$

5090 reflections

108 parameters

4 restraints

Primary atom site location: structure-invariant direct methods
20238 measured reflections

5090 independent reflections

3409 reflections with $I>2 \sigma(I)$

$R_{\text {int }}=0.061$

$\theta_{\text {max }}=39.0^{\circ}, \theta_{\min }=2.3^{\circ}$

$h=-34 \rightarrow 35$

$k=-16 \rightarrow 16$

$l=-18 \rightarrow 14$

Secondary atom site location: difference Fourier map

Hydrogen site location: inferred from neighbouring sites

$\mathrm{H}$ atoms treated by a mixture of independent and constrained refinement

$w=1 /\left[\sigma^{2}\left(F_{\mathrm{o}}^{2}\right)+(0.0666 P)^{2}\right]$ where $P=\left(F_{\mathrm{o}}^{2}+2 F_{\mathrm{c}}{ }^{2}\right) / 3$

$(\Delta / \sigma)_{\max }=0.007$

$\Delta \rho_{\max }=0.90 \mathrm{e} \AA^{-3}$

$\Delta \rho_{\min }=-1.44$ e $\AA^{-3}$

\section{Special details}

Geometry. All e.s.d.'s (except the e.s.d. in the dihedral angle between two 1.s. planes) are estimated using the full covariance matrix. The cell e.s.d.'s are taken into account individually in the estimation of e.s.d.'s in distances, angles and torsion angles; correlations between e.s.d.'s in cell parameters are only used when they are defined by crystal symmetry. An approximate (isotropic) treatment of cell e.s.d.'s is used for estimating e.s.d.'s involving 1.s. planes.

Refinement. Refinement of $F^{2}$ against ALL reflections. The weighted $R$-factor $w R$ and goodness of fit $S$ are based on $F^{2}$, conventional $R$-factors $R$ are based on $F$, with $F$ set to zero for negative $F^{2}$. The threshold expression of $F^{2}>\sigma\left(F^{2}\right)$ is used only for calculating $R$-factors(gt) etc. and is not relevant to the choice of reflections for refinement. $R$-factors based on $F^{2}$ are statistically about twice as large as those based on $F$, and $R$ - factors based on ALL data will be even larger.

Fractional atomic coordinates and isotropic or equivalent isotropic displacement parameters $\left(\AA^{2}\right)$

\begin{tabular}{lllll}
\hline & $x$ & $y$ & $z$ & $U_{\text {iso }}^{*} / U_{\text {eq }}$ \\
\hline Co1 & 0.5 & $0.47467(3)$ & 0.25 & $0.01473(8)$ \\
S1 & $0.76287(2)$ & $0.53220(4)$ & $0.58938(4)$ & $0.01334(9)$ \\
C11 & $0.43290(2)$ & $0.30294(4)$ & $0.04608(4)$ & $0.01647(9)$ \\
O1W & $0.59916(7)$ & $0.49821(15)$ & $0.22088(14)$ & $0.0177(2)$ \\
H1W & $0.5953(15)$ & $0.556(2)$ & $0.168(2)$ & $0.027^{*}$ \\
H2W & $0.6412(11)$ & $0.483(3)$ & $0.288(2)$ & $0.027^{*}$ \\
O2W & $0.54617(7)$ & $0.65037(13)$ & $0.41295(13)$ & $0.0155(2)$ \\
H3W & $0.5200(12)$ & $0.669(3)$ & $0.452(2)$ & $0.023^{*}$ \\
H4W & $0.5887(12)$ & $0.631(3)$ & $0.467(3)$ & $0.023^{*}$ \\
C3 & $0.90875(11)$ & $0.5145(2)$ & $0.7520(3)$ & $0.0298(5)$ \\
H3A & 0.955 & 0.5538 & 0.8296 & $0.036^{*}$ \\
H3B & 0.9206 & 0.4724 & 0.6815 & $0.036^{*}$ \\
C4 & $0.84886(10)$ & $0.6288(2)$ & $0.6830(2)$ & $0.0240(4)$ \\
H4A & 0.8584 & 0.686 & 0.6172 & $0.029^{*}$ \\
H4B & 0.8475 & 0.69 & 0.7554 & $0.029^{*}$
\end{tabular}


supporting information

\begin{tabular}{lllll} 
C1 & $0.79391(13)$ & $0.3742(2)$ & $0.6942(2)$ & $0.0293(4)$ \\
H1A & 0.7618 & 0.3529 & 0.737 & $0.035^{*}$ \\
H1B & 0.7924 & 0.2945 & 0.635 & $0.035^{*}$ \\
C2 & $0.87487(14)$ & $0.4035(3)$ & $0.8102(3)$ & $0.0386(6)$ \\
H2A & 0.9047 & 0.317 & 0.8342 & $0.046^{*}$ \\
H2B & 0.8752 & 0.4386 & 0.8966 & $0.046^{*}$ \\
O12 & $0.70254(8)$ & $0.60180(16)$ & $0.60336(16)$ & $0.0245(3)$ \\
O11 & $0.74827(8)$ & $0.50189(17)$ & $0.44424(16)$ & $0.0273(3)$ \\
\hline
\end{tabular}

Atomic displacement parameters $\left(\AA^{2}\right)$

\begin{tabular}{lllllll}
\hline & $U^{11}$ & $U^{22}$ & $U^{33}$ & $U^{12}$ & $U^{13}$ & $U^{23}$ \\
\hline Co1 & $0.01483(14)$ & $0.01815(15)$ & $0.01223(15)$ & 0 & $0.00731(12)$ & 0 \\
S1 & $0.01081(15)$ & $0.01611(17)$ & $0.01057(17)$ & $-0.00112(12)$ & $0.00313(13)$ & $0.00104(12)$ \\
C11 & $0.02063(18)$ & $0.01729(17)$ & $0.01137(16)$ & $-0.00469(13)$ & $0.00760(14)$ & $-0.00251(13)$ \\
O1W & $0.0121(5)$ & $0.0302(7)$ & $0.0117(5)$ & $0.0022(4)$ & $0.0064(4)$ & $0.0051(5)$ \\
O2W & $0.0149(5)$ & $0.0186(5)$ & $0.0130(5)$ & $-0.0020(4)$ & $0.0067(4)$ & $-0.0022(4)$ \\
C3 & $0.0136(7)$ & $0.0444(12)$ & $0.0283(11)$ & $0.0051(7)$ & $0.0074(7)$ & $-0.0038(9)$ \\
C4 & $0.0173(7)$ & $0.0219(8)$ & $0.0277(9)$ & $-0.0067(6)$ & $0.0068(7)$ & $-0.0036(7)$ \\
C1 & $0.0397(12)$ & $0.0158(8)$ & $0.0332(11)$ & $0.0009(7)$ & $0.0182(10)$ & $0.0080(7)$ \\
C2 & $0.0326(11)$ & $0.0517(14)$ & $0.0278(11)$ & $0.0189(10)$ & $0.0115(9)$ & $0.0219(10)$ \\
O12 & $0.0160(6)$ & $0.0326(7)$ & $0.0230(7)$ & $0.0034(5)$ & $0.0079(5)$ & $-0.0044(5)$ \\
O11 & $0.0188(6)$ & $0.0506(9)$ & $0.0112(6)$ & $-0.0003(6)$ & $0.0060(5)$ & $-0.0021(6)$ \\
\hline
\end{tabular}

Geometric parameters $\left(\AA,{ }^{\circ}\right)$

\begin{tabular}{llll}
\hline $\mathrm{Co} 1-\mathrm{O} 1 \mathrm{~W}$ & $2.1660(13)$ & $\mathrm{O} 2 \mathrm{~W}-\mathrm{H} 4 \mathrm{~W}$ & $0.79(2)$ \\
$\mathrm{Co1}-\mathrm{O} 1 \mathrm{~W}^{\mathrm{i}}$ & $2.1660(13)$ & $\mathrm{C} 3-\mathrm{C} 4$ & $1.515(3)$ \\
$\mathrm{C} 1-\mathrm{O} 2 \mathrm{~W}$ & $2.2455(12)$ & $\mathrm{C} 3-\mathrm{C} 2$ & $1.529(4)$ \\
$\mathrm{Co1}-\mathrm{O} 2 \mathrm{~W}^{\mathrm{i}}$ & $2.2455(12)$ & $\mathrm{C} 3-\mathrm{H} 3 \mathrm{~A}$ & 0.97 \\
$\mathrm{Co1}-\mathrm{C} 11$ & $2.5102(5)$ & $\mathrm{C} 3-\mathrm{H} 3 \mathrm{~B}$ & 0.97 \\
$\mathrm{C} 1-\mathrm{C} 11^{\mathrm{i}}$ & $2.5102(5)$ & $\mathrm{C} 4-\mathrm{H} 4 \mathrm{~A}$ & 0.97 \\
$\mathrm{~S} 1-\mathrm{O} 12$ & $1.4456(14)$ & $\mathrm{C} 4-\mathrm{H} 4 \mathrm{~B}$ & 0.97 \\
$\mathrm{~S} 1-\mathrm{O} 11$ & $1.4478(16)$ & $\mathrm{C} 1-\mathrm{C} 2$ & $1.518(3)$ \\
$\mathrm{S} 1-\mathrm{C} 4$ & $1.7730(18)$ & $\mathrm{C} 1-\mathrm{H} 1 \mathrm{~A}$ & 0.97 \\
$\mathrm{~S} 1-\mathrm{C} 1$ & $1.7820(19)$ & $\mathrm{C} 1-\mathrm{H} 1 \mathrm{~B}$ & 0.97 \\
$\mathrm{O} 1 \mathrm{~W}-\mathrm{H} 1 \mathrm{~W}$ & $0.758(16)$ & $\mathrm{C} 2-\mathrm{H} 2 \mathrm{~A}$ & 0.97 \\
$\mathrm{O} 1 \mathrm{~W}-\mathrm{H} 2 \mathrm{~W}$ & $0.812(17)$ & $\mathrm{C} 2-\mathrm{H} 2 \mathrm{~B}$ & 0.97 \\
$\mathrm{O} 2 \mathrm{~W}-\mathrm{H} 3 \mathrm{~W}$ & $0.830(16)$ & & \\
& & & $115(2)$ \\
$\mathrm{O} 1 \mathrm{~W}-\mathrm{Co1}-\mathrm{O} 1 \mathrm{~W}^{\mathrm{i}}$ & $168.24(8)$ & $\mathrm{H} 3 \mathrm{~W}-\mathrm{O} 2 \mathrm{~W}-\mathrm{H} 4 \mathrm{~W}$ & $106.16(18)$ \\
$\mathrm{O} 1 \mathrm{~W}-\mathrm{Co1}-\mathrm{O} 2 \mathrm{~W}$ & $88.05(5)$ & $\mathrm{C} 4-\mathrm{C} 3-\mathrm{C} 2$ & 110.5 \\
$\mathrm{O} 1 \mathrm{~W}-\mathrm{Co} 1-\mathrm{O} 2 \mathrm{~W}$ & $83.27(5)$ & $\mathrm{C} 4-\mathrm{C} 3-\mathrm{H} 3 \mathrm{~A}$ & 110.5 \\
$\mathrm{O} 1 \mathrm{~W}-\mathrm{Co} 1-\mathrm{O} 2 \mathrm{~W}^{\mathrm{i}}$ & $83.27(5)$ & $\mathrm{C} 2-\mathrm{C} 3-\mathrm{H} 3 \mathrm{~A}$ & 110.5 \\
$\mathrm{O} 1 \mathrm{~W}-\mathrm{Co} 1-\mathrm{O} 2 \mathrm{~W}^{\mathrm{i}}$ & $88.05(5)$ & $\mathrm{C} 4-\mathrm{C} 3-\mathrm{H} 3 \mathrm{~B}$ & 110.5 \\
$\mathrm{O} 2 \mathrm{~W}-\mathrm{Co1}-\mathrm{O} 2 \mathrm{~W}^{\mathrm{i}}$ & $84.92(7)$ & $\mathrm{C} 2-\mathrm{C} 3-\mathrm{H} 3 \mathrm{~B}$ & 108.7 \\
$\mathrm{O} 1 \mathrm{~W}-\mathrm{Co1}-\mathrm{C} 11$ & $95.34(4)$ & $\mathrm{H} 3 \mathrm{C}-\mathrm{C} 3-\mathrm{H} 3 \mathrm{~B}$ & $103.73(13)$ \\
$\mathrm{O} 1 \mathrm{~W}-\mathrm{Co1}-\mathrm{C} 11$ & $92.24(4)$ & $\mathrm{C} 3-\mathrm{C} 4-\mathrm{S} 1$ &
\end{tabular}




$\begin{array}{llll}\mathrm{O} 2 \mathrm{~W}-\mathrm{Co} 1-\mathrm{Cl1} & 171.61(4) & \mathrm{C} 3-\mathrm{C} 4-\mathrm{H} 4 \mathrm{~A} & 111 \\ \mathrm{O} 2 \mathrm{~W}-\mathrm{Co} 1-\mathrm{Cl1} & 87.85(3) & \mathrm{S} 1-\mathrm{C} 4-\mathrm{H} 4 \mathrm{~A} & 111 \\ \mathrm{O} 1 \mathrm{~W}-\mathrm{C} 1-\mathrm{Cl1} 1^{\mathrm{i}} & 92.24(4) & \mathrm{C} 3-\mathrm{C} 4-\mathrm{H} 4 \mathrm{~B} & 111 \\ \mathrm{O} 1 \mathrm{~W}-\mathrm{Co} 1-\mathrm{Cl1} 1^{\mathrm{i}} & 95.34(4) & \mathrm{S} 1-\mathrm{C} 4-\mathrm{H} 4 \mathrm{~B} & 111 \\ \mathrm{O} 2 \mathrm{~W}-\mathrm{C} 1-\mathrm{C} 11^{\mathrm{i}} & 87.85(3) & \mathrm{H} 4 \mathrm{~A}-\mathrm{C} 4-\mathrm{H} 4 \mathrm{~B} & 109 \\ \mathrm{O} 2 \mathrm{~W}-\mathrm{Co} 1-\mathrm{Cl1} 1^{\mathrm{i}} & 171.61(4) & \mathrm{C} 2-\mathrm{C} 1-\mathrm{S} 1 & 110.63(15) \\ \mathrm{C} 1-\mathrm{Co} 1-\mathrm{Cl} 1^{\mathrm{i}} & 99.66(2) & \mathrm{C} 2-\mathrm{C} 1-\mathrm{H} 1 \mathrm{~A} & 110.6 \\ \mathrm{O} 12-\mathrm{S} 1-\mathrm{O} 11 & 116.50(9) & \mathrm{S} 1-\mathrm{C} 1-\mathrm{H} 1 \mathrm{~A} & 110.6 \\ \mathrm{O} 12-\mathrm{S} 1-\mathrm{C} 4 & 110.32(9) & \mathrm{C} 2-\mathrm{C} 1-\mathrm{H} 1 \mathrm{~B} & 110.6 \\ \mathrm{O} 11-\mathrm{S} 1-\mathrm{C} 4 & 109.92(10) & \mathrm{S} 1-\mathrm{C} 1-\mathrm{H} 1 \mathrm{~B} & 108.7 \\ \mathrm{O} 12-\mathrm{S} 1-\mathrm{C} 1 & 112.01(10) & \mathrm{H} 1 \mathrm{~A}-\mathrm{C} 1-\mathrm{H} 1 \mathrm{~B} & 107.97(17) \\ \mathrm{O} 11-\mathrm{S} 1-\mathrm{C} 1 & 109.14(10) & \mathrm{C} 1-\mathrm{C} 2-\mathrm{C} 3 & 110.1 \\ \mathrm{C} 4-\mathrm{S} 1-\mathrm{C} 1 & 97.26(10) & \mathrm{C} 1-\mathrm{C} 2-\mathrm{H} 2 \mathrm{~A} & 110.1 \\ \mathrm{Co} 1-\mathrm{O} 1 \mathrm{~W}-\mathrm{H} 1 \mathrm{~W} & 114(2) & \mathrm{C} 3-\mathrm{C} 2-\mathrm{H} 2 \mathrm{~A} & 110.1 \\ \mathrm{C} 1-\mathrm{O} 1 \mathrm{~W}-\mathrm{H} 2 \mathrm{~W} & 120(2) & \mathrm{C} 1-\mathrm{C} 2-\mathrm{H} 2 \mathrm{~B} & 108.4 \\ \mathrm{H} 1 \mathrm{~W}-\mathrm{O} 1 \mathrm{~W}-\mathrm{H} 2 \mathrm{~W} & 118(3) & \mathrm{C} 3-\mathrm{C} 2-\mathrm{H} 2 \mathrm{~B} & \\ \mathrm{C} 1-\mathrm{O} 2 \mathrm{~W}-\mathrm{H} 3 \mathrm{~W} & 114.7(17) & \mathrm{H} 2 \mathrm{~A}-\mathrm{C} 2-\mathrm{H} 2 \mathrm{~B} & 115.94(17) \\ \mathrm{C} 1-\mathrm{O} 2 \mathrm{~W}-\mathrm{H} 4 \mathrm{~W} & 106.8(18) & & 1.88(19) \\ & & & -27.0(2) \\ \mathrm{C} 2-\mathrm{C} 3-\mathrm{C} 4-\mathrm{S} 1 & -42.0(2) & \mathrm{O} 11-\mathrm{S} 1-\mathrm{C} 1-\mathrm{C} 2 & 45.6(3) \\ \mathrm{O} 12-\mathrm{S} 1-\mathrm{C} 4-\mathrm{C} 3 & 140.20(15) & \mathrm{C} 4-\mathrm{S} 1-\mathrm{C} 1-\mathrm{C} 2 & \\ \mathrm{O} 11-\mathrm{S} 1-\mathrm{C} 4-\mathrm{C} 3 & -89.99(17) & \mathrm{S} 1-\mathrm{C} 1-\mathrm{C} 2-\mathrm{C} 3 & \\ \mathrm{C} 1-\mathrm{S} 1-\mathrm{C} 4-\mathrm{C} 3 & 23.45(17) & \mathrm{C} 4-\mathrm{C} 3-\mathrm{C} 2-\mathrm{C} 1 & \\ \mathrm{O} 12-\mathrm{S} 1-\mathrm{C} 1-\mathrm{C} 2 & -113.53(17) & & \end{array}$

Symmetry code: (i) $-x+1, y,-z+1 / 2$.

Hydrogen-bond geometry $\left(\AA,{ }^{\circ}\right)$

\begin{tabular}{lllll}
\hline$D-\mathrm{H} \cdots A$ & $D-\mathrm{H}$ & $\mathrm{H} \cdots A$ & $D \cdots A$ & $D-\mathrm{H} \cdots A$ \\
\hline $\mathrm{O} 1 W-\mathrm{H} 1 W \cdots \mathrm{C} 11^{\mathrm{ii}}$ & $0.77(3)$ & $2.44(3)$ & $3.1885(15)$ & $165(3)$ \\
$\mathrm{O} 1 W-\mathrm{H} 2 W \cdots \mathrm{O} 11$ & $0.81(2)$ & $1.99(2)$ & $2.782(2)$ & $165(2)$ \\
$\mathrm{O} 2 W-\mathrm{H} 3 W \cdots \mathrm{C} 11^{\mathrm{iii}}$ & $0.83(2)$ & $2.41(2)$ & $3.2289(16)$ & $171(2)$ \\
$\mathrm{O} 2 W-\mathrm{H} 4 W \cdots \mathrm{O} 12$ & $0.79(3)$ & $2.05(3)$ & $2.835(2)$ & $174(3)$
\end{tabular}

Symmetry codes: (ii) $-x+1,-y+1,-z$; (iii) $x,-y+1, z+1 / 2$. 